International Journal of Quantum Information

Vol. 15, No. 3 (2017) 1730001 (18 pages)

(C) The Author(s)

DOI: $10.1142 /$ S0219749917300017

\title{
Quantum image processing: A review of advances in its security technologies
}

\author{
Fei Yan* \\ School of Computer Science and Technology, \\ Changchun University of Science and Technology, \\ No. 7089, Weixing Road, Changchun 130022, China \\ yanfei@cust.edu.cn \\ Abdullah M. Iliyasu \\ Department of Electrical Engineering, College of Engineering, \\ Prince Sattam Bin Abdulaziz University, \\ Al-Kharj 11942, Kingdom of Saudi Arabia \\ a.iliyasu@psau.edu.sa \\ Phuc Q. Le \\ NetMile Inc., 2-4 Kojimachi, \\ Chiyoda-ku, Tokyo 102-0083, Japan \\ phuclevn@gmail.com \\ Received 20 May 2016 \\ Revised 22 November 2016 \\ Accepted 6 March 2017 \\ Published 3 April 2017
}

\begin{abstract}
In this review, we present an overview of the advances made in quantum image processing (QIP) comprising of the image representations, the operations realizable on them, and the likely protocols and algorithms for their applications. In particular, we focus on recent progresses on QIP-based security technologies including quantum watermarking, quantum image encryption, and quantum image steganography. This review is aimed at providing readers with a succinct, yet adequate compendium of the progresses made in the QIP sub-area. Hopefully, this effort will stimulate further interest aimed at the pursuit of more advanced algorithms and experimental validations for available technologies and extensions to other domains.
\end{abstract}

Keywords: Quantum image; quantum movie; quantum audio; quantum watermarking; quantum image encryption; quantum image steganography.

\footnotetext{
${ }^{*}$ Corresponding author.

This is an Open Access article published by World Scientific Publishing Company. It is distributed under the terms of the Creative Commons Attribution 4.0 (CC-BY) License. Further distribution of this work is permitted, provided the original work is properly cited.
} 


\section{Introduction}

Quantum image processing (QIP) is an emerging sub-discipline that is focused on extending conventional image processing tasks and operations to the quantum computing framework. ${ }^{1}$ It is primarily devoted to utilizing quantum computing technologies to capture, manipulate, and recover quantum images in different formats and for different purposes. ${ }^{2}$ Due to some of the astounding properties inherent to quantum computation, notably entanglement and parallelism, it is anticipated that QIP technologies will offer capabilities and performances that are, as yet, unrivaled by their traditional equivalents. These improvements could be in terms of computing speed, guaranteed security, and minimal storage requirements, etc.

Retrospectively, the first published material relating quantum mechanics to image processing can be traced to Vlasov's work, ${ }^{3}$ in 1997, which focused on the use of a quantum system to recognize orthogonal images. This was followed by efforts using quantum algorithms to search specific patterns in binary images ${ }^{4}$ and detect the posture of certain targets. ${ }^{5}$ Notably, more optics-based interpretation for quantum imaging were initially experimentally demonstrated in Ref. 6 and formalized in Ref. 7 after seven years. However, the pioneering role of research that gave birth to what is today referred to as QIP $^{1}$ should be attributed to Venegas-Andraca and Bose's Qubit Lattice $^{8}$ description for quantum images in 2003. Following this, Lattorre proposed another kind of representation, called the Real Ket, ${ }^{9}$ whose purpose was to encode quantum images as a basis for further applications in QIP.

Technically, these pioneering efforts with the subsequent studies related to them can be classified into three main groups, of which (1) and (2) are deemed outside the purview of this review:

(1) Quantum-assisted digital image processing: These applications aim at exploiting some of the properties responsible for the potency of quantum computing algorithms in order to improve some well-known digital or classical image processing tasks and applications. ${ }^{1,2}$

(2) Optics-based quantum imaging: These applications focus on devising novel techniques for optical imaging and parallel information processing at the quantum level by exploiting the quantum nature of light and the intrinsic parallelism of optical signals. ${ }^{10}$

(3) Classically-inspired QIP: These applications derive their inspiration from the expectation that quantum computing hardware will soon be physically realized and, hence, such research focuses on extending classical image processing tasks and applications to quantum computing framework. ${ }^{1,2}$

The innovative representations ${ }^{8,9}$ to encode images on the quantum computing framework set the stage for Le et al. to propose the FRQI ${ }^{11}$ (i.e. flexible representation for quantum images) that truly kick-started research in the context of present day descriptions of QIP. This could be attributed to the flexible manner it supports the integration of the quantum image into a normalized state, which facilitates 
auxiliary transformations on the content of the image. Since the FRQI, many other quantum image representations (QIRs) have been proposed as well as an array of algorithmic frameworks that target the spatial or chromatic content of the image. ${ }^{2}$

Among others, the multi-channel representation for quantum images (MCQI) ${ }^{12}$ and novel enhanced quantum image representation (NEQR) ${ }^{13}$ are the representative QIRs that have traced their root to the FRQI representation. In addition, a lot of the effort in QIP has been focused on designing algorithms to manipulate the position and color information encoded using the FRQI and its many variants. For instance, FRQIbased fast geometric transformations including (two-point) swapping, flip, (orthogonal) rotations ${ }^{14}$ and restricted geometric transformations to constrain these operations to a specified area of an image ${ }^{15,16}$ were initially proposed. Recently, NEQR-based quantum image translation to map the position of each picture element in an input image into a new position in an output image ${ }^{17}$ and quantum image scaling to resize a quantum image $^{18}$ were discussed. While FRQI-based general form of color transformations were first proposed by means of the single qubit gates such as $\mathrm{X}, \mathrm{Z}$, and $\mathrm{H}$ gates. ${ }^{19}$ Later, MCQI-based channel of interest (CoI) operator to entail shifting the grayscale value of the preselected color channel and the channel swapping (CS) operator to swap the grayscale values between two channels were fully discussed in Ref. 12 .

To illustrate the feasibility and capability of QIP algorithms and application, researchers always prefer to simulate the digital image processing tasks on the basis of the QIRs that we already have. By using the basic quantum gates and the aforementioned operations, so far, researchers have contributed to quantum image feature extraction, ${ }^{20}$ quantum image segmentation, ${ }^{21}$ quantum image morphology, ${ }^{22}$ quantum image comparison, ${ }^{23,24}$ among others. In particular, QIP-based security technologies have attracted extensive interest of researchers as presented in the ensuing discussions.

As mentioned earlier, the main objectives of this review are twofold: (1) to provide readers with a succinct, yet adequate compendium on QIP and technologies realizable based on it; and (2) to accentuate the advances made in QIP as a motivation to pursue their improvement and experimental validation. To meet these objectives, the review is outlined as follows: the various QIRs and other quantum signal processing concepts that they have inspired are reviewed in Sec. 2. Following that, some of the outstanding QIP-based security technologies are elucidated in Sec. 3. Finally, we present a stimulating discussion regarding the state of QIP research and expected progress over the next few years, especially considering the anticipated realization of physical quantum computing hardware.

\section{Quantum Representations of Image, Movie, and Audio}

This section highlights the various QIRs, including their properties and how they differ from one another. Also, we will dwell on how these representations have inspired formulations for other essential signal processing, i.e. quantum movie and quantum audio. 


\subsection{Quantum image representation}

Inspired by the classical (digital) representation of an image, an $N$ pixel sized quantum image consists of two parts that capture information about color and position about every stiver that makes up the image. The general representation for a quantum image is presented as:

$$
|I\rangle=\frac{1}{\sqrt{N}} \sum_{p=0}^{N-1}\left|C_{p}\right\rangle \otimes|P\rangle,
$$

where $\left|C_{p}\right\rangle$ represents the color information of a quantum image and $|P\rangle$ indicates the corresponding position information. With the tensor product $(\otimes)$, the information about an image can be integrated into a normalized quantum state. Considering the widely used two-dimensional Cartesian coordinate system,

$$
|P\rangle=|y\rangle|x\rangle=\left|y_{n-1} y_{n-2} \ldots y_{0}\right\rangle\left|x_{n-1} x_{n-2} \ldots x_{0}\right\rangle,
$$

wherein $x_{j}, y_{j} \in\{0,1\}$. In order to manipulate a quantum image information for different purposes, various studies focus on improvements that have been suggested for the chromatic components encoding the image. In this review, we will concentrate on the three frequently-used QIRs.

\subsubsection{Flexible representation for quantum images-FRQI}

In 2011, Le et al. proposed the FRQI, ${ }^{11}$ which has since burgeoned into the most widely used QIR. The FRQI captures the essential information about the colors as well as the corresponding positions of every point in an image and integrates them into a normalized quantum state. In particular, it uses a vector of angles to encode color information in the form:

$$
\left|C_{f}\right\rangle=\cos \theta_{f}|0\rangle+\sin \theta_{f}|1\rangle
$$

where $|0\rangle$ and $|1\rangle$ are $2 \mathrm{D}$ computational basis quantum states, $\theta_{f} \in\left[0, \frac{\pi}{2}\right]$, and $f=0,1,2, \ldots, 2^{2 n}-1$. The polynomial preparation theorem (PPT) as developed by using Theorem 1 in Ref. 11 shows a constructively efficient implementation of the FRQI preparation process. Therein, the computational complexity of the whole preparation shows that an FRQI image state requires $O\left(2^{4 n}\right) \cdot{ }^{11}$

\subsubsection{Multi-channel representation for quantum images-MCQI}

By extending the grayscale information encoded in an FRQI image to a color representation, MCQI, which uses R, G, and B channels to represent the different color informations about the image while also retaining its normalized state, was proposed in Ref. 12. An MCQI representation is realized by assigning three qubits to encode color information about the images:

$$
\begin{aligned}
\left|C_{m}\right\rangle= & \cos \theta_{R}^{m}|000\rangle+\cos \theta_{G}^{m}|001\rangle+\cos \theta_{B}^{m}|010\rangle+\cos \theta_{\alpha}|011\rangle \\
& +\sin \theta_{R}^{m}|100\rangle+\sin \theta_{G}^{m}|101\rangle+\sin \theta_{B}^{m}|110\rangle+\sin \theta_{\alpha}|111\rangle,
\end{aligned}
$$


where $\left\{\theta_{R}^{m}, \theta_{G}^{m}, \theta_{B}^{m}\right\} \in[0, \pi / 2]$ are three angles encoding the colors of the $\mathrm{R}, \mathrm{G}$, and $\mathrm{B}$ channels of the $m$ th pixel, respectively. Based on the MCQI representation, all RGB information about an image is stored simultaneously.

MCQI representation provides a strategy to encode $R, G$, and B channel information in normalized quantum states using many fewer qubits. Using MCQI representation makes it easier to design color image operators with lower complexity. In addition, MCQI representation provides the potential to design quantum-cryptography-based color image watermarking algorithms. An MCQI image is stored in the preparation process using the multi-channel PPT (MC-PPT) theorem ${ }^{12}$ that extends the vector in (FRQI) PPT to three vectors of angles. The computational complexity of the whole preparation for MCQI image is the same as that required for an FRQI image, i.e. $O\left(2^{4 n}\right) .{ }^{12}$

\subsubsection{Novel enhanced quantum image representation-NEQR}

Zhang et al.'s NEQR ${ }^{13}$ representation for digital images is based on extending the storage of the grayscale value of every pixel instead of an angle encoded in a qubit in FRQI to the basis state of a qubit sequence. Therefore, to store the digital image in NEQR representation using quantum mechanics, two entangled qubit sequences are used to store the whole image, which represent the grayscale and positional information of all the pixels. Suppose the grayscale range of an image is $2^{q}$ with a binary sequence $C_{y x}^{q-1} C_{y x}^{q-2} \cdots C_{y x}^{0}$ encoding the grayscale value as:

$$
\left|C_{n}\right\rangle=\left|C_{n}^{q-1} C_{n}^{q-2} \cdots C_{n}^{0}\right\rangle,
$$

where $C_{n}^{k} \in\{0,1\}$ and $C_{n} \in\left[0,2^{q}-1\right]$. To prepare an NEQR image, the first step is similar with the preparation of an FRQI image. While the second step is divided into $2^{2 n}$ sub-operations to store the grayscale information for every pixel. ${ }^{13}$

The NEQR representation utilizes the basis state of a qubit sequence to represent the grayscale values of pixels rather than the probability amplitude of a single qubit used in the FRQI representation. Therefore, as claimed in Ref. 13, it could offer significant advantages in image compression, image operations, and image retrieval. They also assert that the computational complexity of preparing the NEQR image exhibits an approximately quadratic decrease, i.e. $O\left(q n \cdot 2^{2 n}\right)$, compared to FRQI.

\subsection{Quantum movie representation}

Inspired by traditional requirements to describe and produce a movie (also called a motion picture, which is essentially a series of still images that are played at a predefined frame transition rate, etc.), in 2012, by using the FRQI representation to encode each frame, Iliyasu et al. proposed a framework to represent and produce movies on quantum computers. ${ }^{25}$ This quantum movie framework advanced earlier descriptions of FRQI by extending it to encode a register of multiple FRQI images, 
also called a strip, comprising of $K=2^{k}$ quantum images as formulated in Eq. (6):

$$
|M\rangle=\frac{1}{2^{k / 2}} \sum_{s=0}^{K-1}\left|F_{s}(n)\right\rangle \otimes|s\rangle,
$$

where $|s\rangle\left(s=0,1, \ldots, 2^{k}-1\right)$ is the position of each image in the strip, $k$ is the number of qubits required to encode the images in the movie, $\left|F_{s}(n)\right\rangle$ is an FRQI image as defined earlier (it is trivial that using Eqs. (1)-(5), the strip representation in Eq. (6) could be extended to encode multiple MCQI and NEQR images as presented in Refs. 26 and 27) at position $|s\rangle$. In addition, as detailed in Ref. 25, the several conceptual devices adopted in the quantum movie framework, i.e. quantum compact disk (CD), quantum player, and movie reader, provide the necessary tools required to prepare, manipulate, and recover the content of a quantum movie. Interested readers are invited to refer to Refs. 25 and 28 for additional details on the quantum movie framework.

It is noteworthy that two major milestones in the development of traditional movies are the transition from black and white screens to full color movies as well as the incorporation of sound into the silent movies that characterized early movie production. The strip representation provides an intuitive way to employ the time components for synchronization between the audio amplitudes and the image strip for the realization of talking quantum movies. As a first step, efforts must be made to represent the audio signals on quantum computers. This is highlighted in the next section.

\subsection{Quantum audio representation}

Although a different signal, the progress recorded in QIP as enumerated in earlier sections of this review and similar ones elsewhere, ${ }^{1,29,2}$ has also inspired research into quantum audio signal processing.

Like images, sound (or audio) is an indispensable multimedia system. Even though its need was first highlighted in Iliyasu et al.'s "silent" quantum movies, ${ }^{25}$ on quantum computers, the notion of quantum audio is only beginning to gain traction now. Recently, Wang made an attempt to kick-start the development of quantum audio processing (QAP) by proposing the quantum representation for digital audio, i.e. QRDA. ${ }^{30}$ While the layout of the QRDA very much resembles the NEQR, it assigns a different physical interpretation for each item. QRDA uses two entangled qubit sequences to store the audio amplitude and time information and then stores the whole digital audio in the superposition of the two qubit sequences with its formulation in the form presented in Eq. (7):

$$
|A\rangle=\frac{1}{2^{l / 2}} \sum_{t=0}^{L-1}\left|D_{t}\right\rangle \otimes|t\rangle,
$$

where $\left|D_{t}\right\rangle=\left|D_{t}^{0} D_{t}^{1} \ldots D_{t}^{q-1}\right\rangle, D_{t}^{i} \in\{0,1\}$, is the binary sequence of the sample amplitude value and $|t\rangle=\left|t_{0} t_{1} \ldots t_{l-1}\right\rangle, t_{i} \in\{0,1\}$, is the corresponding time 
information in the audio. Therefore, QRDA requires $(q+l)$ qubits to represent an $L$ length audio signal with amplitude range $2^{q}$ (where $L$ is the length of the audio, and when $L=2^{l}$, the redundancies in the quantum audio will be avoided ${ }^{30}$ ). Similar to its formulation, the preparation of a QRDA audio signal resembles that of an NEQR image. Readers are referred to Refs. 30 and 31 for more details on the QAP.

\section{Quantum Image Processing based Security Technologies}

Whether from ancient Greek or Chinese myths, the need for secure communication has evolved with mankind's need to transmit information. For example, ancient Greece scytale was used to restore the secret message in the belt; attack warning was written on a wooden panel and covered in wax; and some identified marks were created on the paper by laying a wire sewn onto the paper mold. These are different strategies for satisfying different requirements but all for the secure communications in those special periods.

The advent of digital technology has ushered a heightened need for secure communication. QIP seeks to facilitate a transition from image processing and applications using traditional (digital) computing to the more sophisticated quantum computing paradigm. As highlighted in the opening section of this review, QIP is built entirely on the extension of digital image processing to quantum computing realm leading to the realization of secure, efficient, and advanced technologies for cryptography and information hiding. Indeed, within these two technologies, quantum computation and QIP offer the possibilities for secure communication in areas such as encryption, steganography, and watermarking. Figure 1 provides an outline of quantum image security technologies within these three broad areas. Indeed, the forgoing three stories in ancient times could be regarded as the early rudiments of these three areas.

As a direct application in the science of cryptography, encryption is considered as the process of obscuring information to make it unreadable without special knowledge. $^{32}$ This is usually done for secrecy, and typically for confidential communications. On its part, cryptography is about protecting the content of messages, whereas information hiding is focused on concealing their very existence. ${ }^{33}$ Information hiding using strategies such as steganography and watermarking seems more secure because

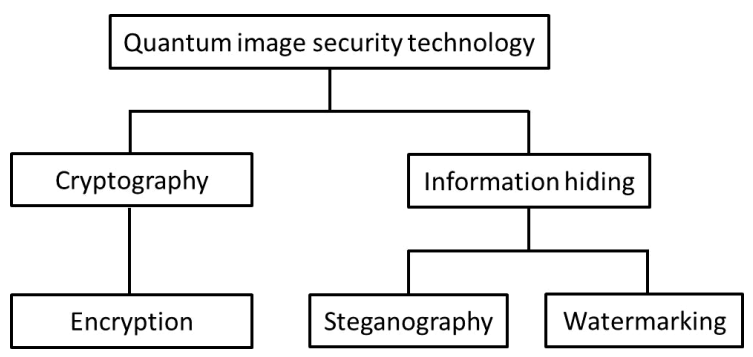

Fig. 1. Outline of quantum image security technologies. 
it is not easily noticed by the attackers. However, among its main constrains is its high demands related to the amount of information that can be hidden inside the cover image without distortions to its visible imperceptibility. While the two strategies (steganography and watermarking) are interrelated, they differ in terms of purpose and/or applications as well as in terms of the requirements for those purposes, which we enumerate in the sequel. ${ }^{33}$

(1) In watermarking, the conspicuous (or visible) content is the carrier image whereas the copyright or ownership is hidden and subject to authentication. In the case of steganography, its objective is to securely communicate the secret message by camouflaging it as a meaningless part of the carrier image without triggering any suspicions from third party adversaries.

(2) In watermarking, information is hidden in the form of a stochastic serial number or some image such as a logo. Therefore, watermarked images usually carry a small amount of information about the copyright ownership. Since the objective of steganography is to camouflage the presence of the hidden message, oftentimes, it requires large carrying capacity in terms of the carrier image.

(3) In watermarking, the watermarked content are susceptible to numerous types of infringements ranging form cropping, filtering, channel noise, etc., which are not of concern in stego images.

The remainder of this section is devoted to reviewing some of the advances recorded in quantum image security protocols that traverse the areas of quantum watermarking, quantum image encryption, and quantum image steganography.

\subsection{Algorithms for quantum watermarking}

Like digital watermarking that was highlighted earlier, quantum watermarking aims to protect the copyright of an image and authenticate its ownership using visible or invisible signals (mostly logos) embedded into the cover (or carrier) image. Figure 2 presents the schematic outlining the approach used in quantum watermarking.

Based on the description of a quantum image and the resulting interpretation of QIP as provided in Sec. 2, particularly, the FRQI representation, the work in Ref. 34 by Iliyasu et al. is credited as being the first quantum image security protocol. In that study, a scheme called WaQI (i.e. watermarking and authentication of quantum images) was proposed based on restricted geometric transformations on the images. Proposed as an invisible watermarking strategy, the WaQI is adjudged as being a secure, keyless, blind, and computationally efficient scheme that can perfectly authenticate ownership of watermarked images. WaQI is built based on using a cover and watermark image pair to produce a watermark embedding circuit that is used to randomly hide the watermark inside the carrier image. This same circuit is reversed to recover the original (unmarked) image during the copyright ownership validation. Following the WaQI scheme, its grayscale version, the gray $\mathrm{WaQI}^{35}$ (or gWaQI) was 


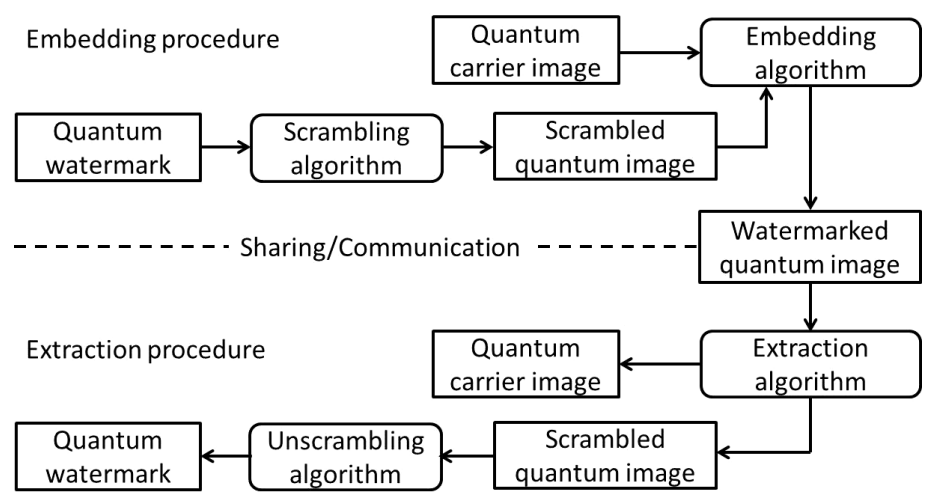

Fig. 2. Schematic for quantum watermarking algorithms.

proposed as a two-tier watermark strategy that facilitates embedding a conspicuous watermark logo in a predetermined sub-area of the cover image; meanwhile, the same watermark signal $(\operatorname{logo})$ is also embedded to cover the rest of the image in an obscure manner.

Although the original WaQI scheme has proven effective in authenticating ownership of watermarked images, its main drawback is that the content of the watermark is required in order to realize the watermark authentication circuit, which is required to validate the ownership of the marked images. Many of the studies that followed were focused on solving this shortcoming of the WaQI scheme. In Ref. 36, the quantum Fourier transform (QFT) was used to extract the watermarking image without having to know what it looks like. However, this approach also had its own drawbacks, since as a result of the computation, the hitherto normalized quantum image state is lost. This is attributed to the fixed embedding strength controlling parameter. $^{36,37}$ In addition, two dynamic watermarking schemes, i.e. quantum Wavelet transform (QWT)-based watermarking ${ }^{38}$ and Hadamard transform-based watermarking ${ }^{39}$ were proposed. Instead of the fixed embedding strength parameter used in Ref. 36, this scheme utilizes dynamic vectors to control the embedding strength. In this manner, a better trade-off between visual quality and embedding capacity is guaranteed so that the scheme improves on previous efforts.

The quantum watermarking strategies reviewed thus far are all based on the FRQI representation for the carrier images and watermark logos. Using the multichannel extension of the FRQI representation, i.e. the MCQI representation, an MCQI extension of the WaQI scheme (the MC-WaQI) was proposed in Ref. 40. In MC-WaQI, two keys, i.e. color information key (CIK) and position information key $(\mathrm{PIK})$, are generated in the preprocessing stage of the scheme. Following this, two watermark images, one each transformed by CIK on the chromatic content and PIK on the spatial content, are embedded into the spatial domain and frequency domain of the cover image, respectively. The adoption of MCQI representation for the carrier 
and watermark images facilitates the protection of colored quantum images and improves the capability of watermarked images to withstand malicious attacks.

In extending quantum watermarking to grayscale images, Ref. 41 proposed the use of simple and small-scale quantum circuits to embed a scrambled image onto the carrier image using CNOT gates (i.e. the exclusive or XOR operation). Simulationbased results presented in the study validated the performance of the proposed scheme in terms of the visual quality, robustness performance, and computational complexity. More recently, in the study presented in Ref. 42, a new watermarking strategy where the carrier and the watermark images are stored in the $\theta$ and $\psi$ phases of the same qubit, respectively. The study claims that visual imperceptibility is guaranteed irrespective of the size of image being embedded, i.e. the embedding capacity of the carrier image.

\subsection{Algorithms for quantum image encryption}

Encryption is pivotal to secure communication and information sharing, especially in warfare, military communication, politics, and even the daily lives of ordinary people as necessitated by the ubiquity of information, notably images and video, sharing and storage.

A few requirements for encryption were highlighted earlier, but we should stress the need for fast computing speed (in real-time) for applications such as video conferencing; high fidelity, for example, in applications such as accurate disease diagnosis in telemedicine; and large processing area due to the strong correlation and high redundancy of image data. In efforts to satisfy some or most of these requirements, quantum image encryption technologies could be broadly classified as being spatial or frequency domain-based strategies as summarized in the schematic presented in Fig. 3.

Information about the spatial domain of a quantum image is also described by its position information (pixel position) and color information (pixel value). Therefore, the algorithms for quantum image encryption targeting such information are mainly focused on these two parameters. The often-used image encryption methods in this regard are scrambling approach (to transform the pixel position to disorder an image) and replacement approach (to transform the pixel value to alter the statistical

Encryption procedure

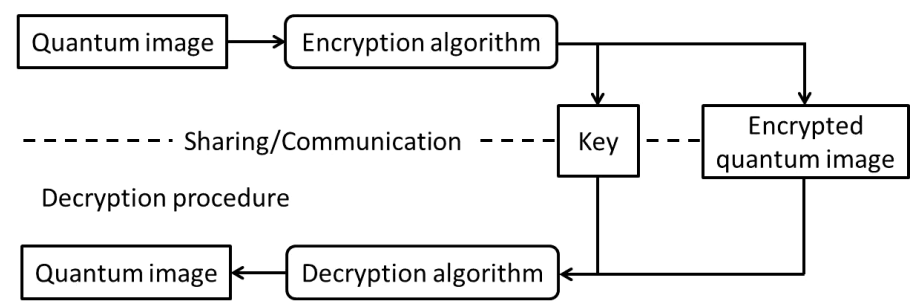

Fig. 3. Schematic for quantum image encryption algorithms. 
property of the encrypted image). Indeed, the combination of these two methods is also an available solution.

Popular scrambling algorithms used on the position information include the Hilbert transform, Arnold transform, and Fibonacci transform. These traditional approaches have been extended to the encryption applications in QIP domain. The Arnold and Fibonacci scrambling quantum circuits in Ref. 43 take advantage of the plain adder and adder modulo $N$ by modifying operations on the input and output in order to scramble the images, while the Hilbert scrambling quantum circuit in Ref. 44 is executed using the Hilbert Scanning Matrix that is generated by a recursive generation algorithm. Preceding this, in Ref. 45, an encryption and decryption algorithm that uses geometric transformations on FRQI images was investigated to reverse the correlations among adjacent pixels but the encrypted images were not noise-like and the sketches can be identified visually.

In addition to the only position space scrambling, algorithms utilizing or combining the replacement approach are also well studied. In 2015, Zhou et al. proposed a quantum image Gray-code and bit-plane scrambling scheme based on NEQR representations. ${ }^{46}$ They reported that the scheme's cost is rather low, and the scrambling speed is very high compared with other quantum image scrambling methods such as the quantum Hilbert scrambling. ${ }^{44}$ Moreover, the encryption scheme in Ref. 47 used geometric transformations to shuffle the codes of pixel positions, and further the color transformations were performed to recode the color codings in the FRQI images. Targeting the RGB-based MCQI image, Ref. 48 introduced a method that applies both color and geometric transformations on an image, where the color information is transformed by quantum rotation operations and the geometric information is scrambled using an improved partition scrambling method, thereby ensuring the security of the quantum images. Finally, a novel quantum image encryption algorithm combining the generalized affine transform with logistic map was studied in Ref. 49. Therein, the gray-level information of the quantum image is encrypted by the XOR operation with a key generator controlled by the logistic map, while the position information of the quantum image is encoded by the generalized affine transform. The proposed algorithm is considered robust with better performance than its classical counterpart in terms of computational complexity. ${ }^{49}$

Turning to discussions on frequency domain-based quantum image encryption protocols, the double random-phase encoding technique is generalized to the quantum computing realm, which was used together with the QFT to realize a robust quantum image encryption method. ${ }^{50}$ Similarly, a novel encryption algorithm for FRQI images based on the QWT and double diffusions was discussed in Ref. 51. In another effort, a quantum encryption and decryption method for color images is proposed, in which QFT and double random-phase encoding techniques have been used as presented in Ref. 52. The encryption process can be realized by performing two secret random-phase encoding operations, respectively, in the input and the QFT planes. Meanwhile, in Ref. 53, a novel quantum image encryption using 
one-dimensional quantum cellular automata, which can be realized by subtly constructing the evolution rules of one-dimensional quantum cellular automata. It is claimed that the algorithm complexity is lower than that of other quantum image encryption schemes based on QFT.

The advances in QIP have inspired the pursuit of studies to extend encryption applications to related media, i.e. quantum movie and quantum audio. With recent efforts related to quantum audio representation and accompanying operations, it seems worthwhile to explore quantum audio encryption. While the sub-area is not as advanced as QIP, it merits mention here that some activity focused on quantum audio and movie encryption ${ }^{54,28}$ is taking place with renewed intensity.

\subsection{Algorithms for quantum image steganography}

As discussed earlier, image steganography is a technique for information hiding focused on concealing a secret message into a carrier image. Figure 4 provides the general schematic for quantum image steganography protocols, while the remainder of this section highlights some advances based on these protocols.

In 2014, in Ref. 55, Jiang et al. proposed a Moiré pattern-based NEQR image steganography strategy. The strategy is designed primarily as a steganographic algorithm with corresponding quantum circuits to hide a binary image into a grayscale image. The embedding algorithm begins with the choice of an initial Moiré grating, i.e. a stochastic image, as the cover image. The initial Moiré grating is then modified according to the secret image, and the deformed Moiré grating is regarded as the Moiré pattern. Finally, the Moiré pattern is altered to obtain the stego image.

Following that study, an enhanced version using two blind LSB (least significant bit) steganography algorithms was proposed in the form of quantum circuits based on NEQR representations. ${ }^{56}$ The first algorithm is anchored on the standard (or plain) LSB, which uses message bits to substitute for the pixels' LSB directly. While the standard LSB steganography system is deemed simple, its robustness is poor. The other is block LSB, which embeds a message bit into a number of pixels that belong to one image block. Block LSB steganography network aims to improve the robustness

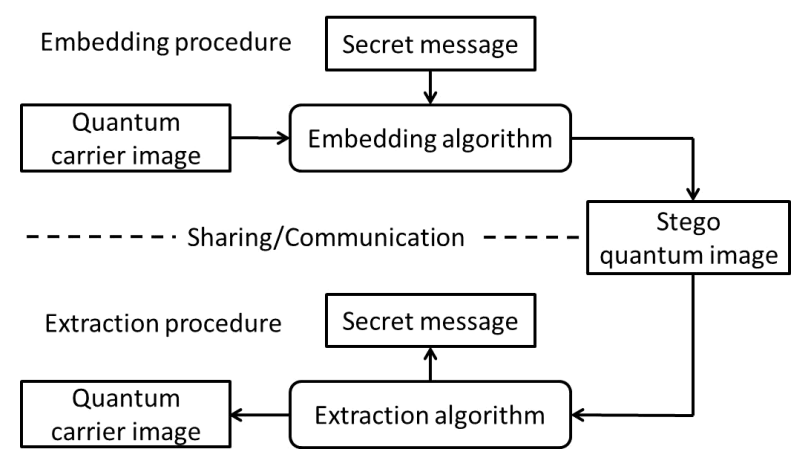

Fig. 4. Schematic for quantum image steganography algorithms. 
and undetectability of standard LSB scheme. This is accomplished by dividing the cover image into blocks and instead of each pixel, each block hides one message bit. In fact, standard LSB steganography can be seen as a special case of block LSB version in which every block can only accommodate one pixel. The experimental results presented in that study (i.e. Ref. 56) demonstrate that the invisibility is good, and the balance between the capacity and the robustness can be adjusted according to the needs of applications.

Independently, elsewhere, a least significant qubit (LSQb) information hiding algorithm for NEQR images was proposed by Wang et al. ${ }^{57}$ LSQb information hiding embeds a secret message qubit stream into the last qubit of the color information of a quantum cover image. In addition to this contribution, to further enhance security, the authors proposed LSQb frequency domain-based information hiding algorithm by executing QFT on the cover image first and then comparing the qubit of color encoding information in secret image with the last qubit of color encoding information in the QFTed cover image using the two qubit quantum comparator. According to the outputs of the quantum comparator circuit, different unitary transformations will facilitate the secret information to be embedded into QFTed cover image.

\section{Discussions}

The notion of quantum computation and quantum information conjures expectations of fast and secure computing technologies, perhaps, this is attributable to the parallelism and entanglement properties integrated into information processing on these paradigms. Therefore, extending digital image processing to the quantum computing realm, i.e. QIP, influences similar expectations.

In this review, we highlighted the recent advances in QIP including the image representations, the algorithms required to operate and manipulate the images, and the likely applications emanating therefrom. Notably, we focused on presenting an overview of QIP-based security technologies.

Le et al.'s FRQI ${ }^{11}$ representation is credited with awakening interest in present day studies in QIP leading to the intensified efforts that have been witnessed recently. Originally, proposed in 2010 and revised a year later, ${ }^{58}$ this study formulated a universally acceptable formulation to represent an image on a quantum computer. The FRQI representation integrates the spatial and chromatic information required to encode an image into a single formula. In this manner, separate or combined transformations to manipulate the content of such images are easily executable.

Following the successful interpretation of a quantum image, there have been many attempts to utilize (for example, Le et al.'s geometric transformation ${ }^{14}$ and color transformation $^{19}$ ); extend (for example, the Sun et al.'s MCQI representation ${ }^{12}$ and Zhang et al.'s NEQR representation ${ }^{13}$ ); or modify (for example, Iliyasu et al.'s quantum movie representation ${ }^{25}$ and Wang's quantum audio representation ${ }^{30}$ ) the FRQI representation for different intents and purposes. Similarly, these advancements have led to many applications in the areas of watermarking, ${ }^{34,36-42}$ 
encryption, ${ }^{43-50,52,59,53}$ and steganography, ${ }^{55-57}$ etc., which form the core security technologies highlighted in this review.

Interestingly, new research groups and individuals are showing interest in this emerging new sub-discipline, i.e. QIP. Recently, research activities in the area emanating from China, Japan, Mexico, and the USA have been increasing, while new ones from Saudi Arabia, India, Iran, and the UK are emerging. The work pursued by these various groups and individuals are focused on expanding the applicability of QIP to realize more classical-like digital image processing algorithms; propose technologies to physically realize the QIP hardware; or simply to note the likely challenges that could impede the realization of some QIP protocols. ${ }^{60}$

The objectives of the discussions presented in this section are also twofold. First, targeting researchers already in the area, a few of the open questions emanating from the published literature are enumerated. The second objective of the discussion is focused mainly on the upcoming researchers that may be interested in pursuing advanced research in the area. In this, our focus is on enumerating some considerations that should guide them in their pursuits. Hopefully, the review presents a compendium of relevant literature needed to invigorate more advanced research in the area.

\subsection{Open questions in $Q I P$}

Within the context of advances made in QIP, there are still a few open questions and areas that need to be improved or expanded. These include:

(1) In addition to the new solutions to the problems considered pertinent, there are open questions pertaining to untouched areas, whose classical equivalents have contributed to the realization of some state-of-the-art image-based applications. Besides enhancing the algorithmic advantages of existing QIP protocols, efforts must be intensified to explore the realization of additional applications such as image registration, mosaic, super-resolution, classification, semantic analysis, reconstruction, denoising, enhancement, fusion, and some possible applications for solving open problems in science and engineering, e.g. computer vision, astrophysics, and medicine, among many others. ${ }^{2}$

(2) Considering the incipient status of the QIP sub-discipline and most of the protocols and applications therefrom, it is important to fashion out benchmarks to ascertain the performance of algorithmic frameworks realizable on the quantum computing realm. For instance, all the QIP algorithms and protocols highlighted in this review rely on the traditional PSNR image quality measure to assess similarity or likeness between images in applications ranging from watermarking, encryption, steganography, etc. As argued in Ref. 24, the quantumness inherent to QIP makes the PSNR image quantity metric a wrong tool to quantify fidelity between quantum images. In proposing a quantum image fidelity metric (QIFM) in Ref. 24, Iliyasu et al. provided the essence for quantum image fidelity 
assessments. However, similar to the advancement of the digital PSNR, more work is still required to improve and concretize its formulation including experimental validations for its use as an image quality metric on quantum computers.

(3) Quantum error correction (QEC) is used to protect quantum information from errors due to decoherence and other quantum noise. ${ }^{61} \mathrm{QEC}$ is essential if one is to achieve fault-tolerant quantum computation that can deal not only with noise on stored quantum information, but also with faulty quantum gates, faulty quantum preparation, and faulty measurements. In QIP, most of the current researches are focused on the image manipulation with very little attention given to the physical preparation and retrieval steps of QIP. These are stages where QEC could make or mar the gains made in the area. It is therefore necessary to consider frameworks for integrating QEC into existing QIP protocols as well as future ones.

(4) On digital computers, images and image processing underpinned advanced applications in videos and movies. Although the quantum movie framework provided a platform to ponder over quantum movie representation and production, since then not much has been added, while it is evident that the concept still requires refinement and expansion. In addition, text, image, and audio represent the main signals, humans are accustomed to using in their day-to-day interactions. While QIP has witnessed tremendous interest and growth, the other two signals (i.e. text and audio) have seen less activity. Recently, however, Wang proposed the quantum representation for digital audio signals. While this is an encouraging effort, like QIP we expect this to be scrutinized and enhanced so that the sub-area of QAP could flourish.

(5) Essentially, QIP is a strategy that using quantum mechanics to store the image information. Indeed, we could consider storing any information by using the similar strategy, besides the information of text, audio, and movie we mentioned above, we can also extend the studies to the representation and manipulation of information in quantum machine learning, quantum neural network, quantum cognition, and quantum cellular automata. Further, when we consider them in a more practical manner, research on the realization of quantum radar, quantum sensor, quantum robot, and quantum nanoscale materials should deserve more attention.

\subsection{Future directions for $Q I P$}

In this sub-section of the review, our discussion is tailored towards stimulating interest for the pursuit of advanced research in QIP by outlining some recommendations that could be adopted for that purpose.

(1) While advanced classical-like QIP technologies are desirable, we must be mindful of the contrariety that permeates information processing in the digital and 
quantum realms. The very properties that are often attributed for the power of quantum computation foreclose the realization of some classical operations. Consequently, in future research efforts, the temptation to realize the quantum version of every digital image processing application that is out there must be resisted. Rather, efforts should be focused in areas and applications where the gains from quantum computation could be exploited. Additionally, since QIP is still in the infant stage, it would enhance the literature if some traditional image processing tasks that could not be accomplished on the quantum computing paradigm because they are in violation of some important postulates should be identified and proven.

(2) No matter how advanced the QIP protocols we realize are, without physical hardware to tangibly utilize them, they will be less meaningful. Thus far, Iliyasu et al.'s exploration on the advances in quantum computation to support the realization of QIP application specific hardware ${ }^{62}$ remains the only attempt to experimentally validate QIP technologies. It is important that we exploit the interdisciplinary nature of quantum computation for improved synergy between researchers with interests in the physics, optics, computer science and engineering aspects of quantum computation to undertake experimental validations of the QIP protocols that are available. As short-term effort, like the transition from analogue to digital computing by using Analog-to-Digital Converter (ADC), it would be expedient to devise a Digital-to-Quantum Converter (DQC) or design a larger device to operate digital and quantum computation side-byside, so that the digital and quantum computing conversion could be seamlessly accomplished.

(3) So far, all so-called experimental implementation of QIP protocols have been restricted to the use of classical computing resources and MATLAB simulations based on linear algebra with complex vectors as quantum states and unitary matrices as unitary transforms. These offer a rather restrictive implementation of the expected power derivable from quantum computation. Therefore, as we intensify efforts in improving and expanding the QIP technologies, it is important to identify the role of quantum computing software required to implement the various algorithms that we have so that they can complement the hardware. Although some effort has been made in terms of quantum software and simulators, such as Refs. 63 and 64, as well as recent interest from the industry such as Refs. 65 and 66, a lot is still required before robust quantum software that can match the advancement in digital image processing are realized. In terms of QIP, it is pertinent to combine insights from computer science and physics to advance present studies aimed at determining which environment is better suited for certain technologies.

It is hoped that the short, mid, and long term directions enumerated in this section would help accelerate efforts towards the realization of more sophisticated QIP-based security technologies. 


\section{Acknowledgments}

This work is supported by the National Natural Science Foundation of China (No. 61502053) and sponsored by the Prince Sattam Bin Abdulaziz University, Saudi Arabia via the Deanship for Scientific Research funding for the CIIS Research group project number 2016/01/6441.

\section{References}

1. A. Iliyasu, Entropy 15 (2013) 2874.

2. F. Yan, A. Iliyasu and S. Venegas-Andraca, Quantum Inf. Process. 15(1) (2016) 1.

3. A. Vlasov, arXiv:quant-ph/9703010.

4. R. Schützhold, Phys. Rev. A 67(6) (2003) 062311.

5. G. Beach, C. Lomont and C. Cohen, Quantum image processing (QUIP), in Proc. 32nd Applied Imagery Pattern Recognition Workshop (2003), pp. 39-44.

6. T. Pittman, Y. Shih, D. Strekalov and A. Sergienko, Phys. Rev. A 52(5) (1995) R3429.

7. L. Lugiato, A. Gatti and E. Brambilla, J. Opt. B 4(3) (2002) S176.

8. S. Venegas-Andraca and S. Bose, Storing, processing, and retrieving an image using quantum mechanics, in Proc. SPIE Conf. Quantum Information and Computation, (2003), pp. 134-147.

9. J. Latorre, arXiv:quant-ph/0510031.

10. A. Gatti, E. Brambilla and L. Lugiato, Prog. Opt. 51(7) (2008) 251.

11. P. Le, F. Dong and K. Hirota, Quantum Inf. Process. 10(1) (2011) 63.

12. B. Sun, A. Iliyasu, F. Yan, F. Dong and K. Hirota, J. Adv. Comput. Intell. Intell. Inf. $\mathbf{1 7}(3)(2013) 404$.

13. Y. Zhang, K. Lu, Y. Gao and M. Wang, Quantum Inf. Process. 12(8) (2013) 2833.

14. P. Le, A. Iliyasu, F. Dong and K. Hirota, IAENG Int. J. Appl. Math. 40(3) (2010) 113.

15. A. Iliyasu, P. Le, F. Dong and K. Hirota, Restricted geometic transformations and their applications for quantum image watermarking and authentications, in Proc. 10th Asian Conf. Quantum Information Sciences (2010), pp. 96-97.

16. P. Le, A. Iliyasu, F. Dong and K. Hirota, Theor. Comput. Sci. 412(15) (2011) 1406.

17. J. Wang, N. Jiang and L. Wang, Quantum Inf. Process. 14(5) (2015) 1589.

18. N. Jiang, J. Wang and Y. Mu, Quantum Inf. Process. 14(11) (2015) 4001.

19. P. Le, A. Iliyasu, F. Dong and K. Hirota, J. Adv. Comput. Intell. Intell. Inf. 15(6) (2011) 698.

20. Y. Zhang, K. Lu, K. Xu, Y. Gao and R. Wilson, Quantum Inf. Process. 14(5) (2015) 1573.

21. S. Caraiman and V. Manta, Theor. Comput. Sci. 529 (2014) 46.

22. S. Yuan, X. Mao, T. Li, Y. Xue, L. Chen and Q. Xiong, Quantum Inf. Process. 14(5) (2015) 1625 .

23. F. Yan, A. Iliyasu, P. Le, B. Sun, F. Dong and K. Hirota, Int. J. Innov. Comput. Appl. 5(4) (2013) 199.

24. A. Iliyasu, F. Yan and K. Hirota, Entropy 18(10) (2016) 360.

25. A. Iliyasu, P. Le, F. Dong and K. Hirota, Int. J. Quantum Inf. 9(6) (2011) 1459.

26. F. Yan, A. Iliyasu, A. Khan and H. Yang, Int. J. Theor. Phys. 55(4) (2016) 2162.

27. F. Yan, A. Iliyasu, K. Hirota and H. Yang, Sci. China Inf. Sci. 59(052102) (2016) 1.

28. F. Yan, A. Iliyasu, S. Venegas-Andraca and H. Yang, Int. J. Theor. Phys. 54(8) (2015) 2893.

29. F. Yan, A. Iliyasu and Z. Jiang, Entropy 16(10) (2014) 5290.

30. J. Wang, Int. J. Theor. Phys. 55(3) 1622 (2016).

31. F. Yan, Y. Guo, A. Iliyasu and H. Yang, arXiv:1701.01291. 
32. M. Walker, Cryptography and encryption overview, CEH Certified Ethical Hacker All-inOne Exam Guide (2011) (McGraw-Hill Ocborne Media).

33. S. Katzenbeisser and F. Petitcolas, Information Hiding Techniques for Steganography and Digital Watermarking (Artech House Print on Demand, 1999)

34. A. Iliyasu, P. Le, F. Dong and K. Hirota, Inf. Sci. 186(1) (2012) 126.

35. A. Iliyasu, P. Le, F. Yan, B. Sun, J. Garcia, F. Dong and K. Hirota, Int. J. Innov. Comput. Appl. 5(2) (2013) 85.

36. W. Zhang, F. Gao, B. Liu, Q. Wen and H. Chen, Quantum Inf. Process. 12(2) (2013) 793.

37. W. Zhang, F. Gao, B. Liu and H. Jia, Int. J. Theor. Phys. 52(2) (2013) 504.

38. X. Song, S. Wang, S. Liu, A. Abd El-Latif and X. Niu, Quantum Inf. Process. 12(2) (2013) 3689.

39. X. Song, S. Wang, A. Abd El-Latif and X. Niu, Multimedia Syst. 20(4) (2014) 379.

40. F. Yan, A. Iliyasu, B. Sun, S. Venegas-Andraca, F. Dong and K. Hirota, Quantum Inf. Process. 14(5) (2015) 1675.

41. S. Miyake and K. Nakamae, Quantum Inf. Process. 15(5) (2016) 1849.

42. P. Li, H. Xiao and B. Li, Quantum Inf. Process. 15 (2016) 4415.

43. N. Jiang, W. Wu and L. Wang, Quantum Inf. Process. 13(5) (2014) 1223.

44. N. Jiang, L. Wang and W. Wu, Int. J. Theor. Phys. 53(7) (2014) 2463.

45. R. Zhou, Q. Wu, M. Zhang and C. Shen, Int. J. Theor. Phys. 52(6) (2013) 1802.

46. R. Zhou, Y. Sun and P. Fan, Quantum Inf. Process. 14(5) (2014) 1717.

47. X. Song, S. Wang, A. Abd El-Latif and X. Niu, Quantum Inf. Process. 13(8) (2014) 1765.

48. F. Yan, Y. Guo, A. Iliyasu, Z. Jiang and H. Yang, J. Adv. Comput. Intell. Intell. Inf. 20(1) (2016) 163.

49. H. Liang, X. Tao and N. Zhou, Quantum Inf. Process. 15 (2016) 2701.

50. Y. Yang, J. Xia, X. Jia and H. Zhang, Quantum Inf. Process. 12(11) (2013) 3477.

51. S. Wang, X. Song and X. Niu, A novel encryption algorithm for quantum images based on quantum wavelet transform and diffusion, Intelligent Data Analysis and its Applications, Volume II, Advances in Intelligent Systems and Computing, Vol. 298 (2014), pp. 243-250. (Springer International Publishing).

52. Y. Yang, X. Jia, S. Sun and Q. Pan, Inf. Sci. 277 (2014) 445.

53. Y. Yang, J. Tian, H. Lei, Y. Zhou and W. Shi, Inf. Sci. 345 (2016) 257.

54. Y. Yang, T. J., S. Sun and P. Xu, Optik 126(21) (2015) 3221.

55. N. Jiang and L. Wang, Int. J. Theor. Phys. 54(3) (2015) 1021.

56. N. Jiang, N. Zhao and L. Wang, Int. J. Theor. Phys. 55(1) (2016) 107.

57. S. Wang, J. Sang, X. Song and X. Niu, Measurement 73 (2015) 352.

58. P. Le, A. Iliyasu, F. Dong and K. Hirota, A flexible representation and invertible transformations for images on quantum computers, New Advances in Intelligent Signal Processing: Studies in Computational Intelligence, Vol. 372 (2011), pp. 179-202. (Springer Berlin Heidelberg).

59. T. Hua, J. Chen, D. Pei, W. Zhang and N. Zhou, Int. J. Theor. Phys. 54(2) (2015) 526.

60. M. Mastriani, arXiv:1512.02942.

61. M. Nielsen and I. Chuang, Quantum Computation and Quantum Information (Cambridge University Press, United Kingdom, 2000).

62. A. Iliyasu, P. Le, F. Yan, B. Sun, J. Garcia, F. Dong and K. Hirota, Int. J. Unconv. Comput. 9(1-2) (2013) 125.

63. D. Wecker and K. Svore, arXiv:1402.4467v1.

64. List of QC simulators, https://quantiki.org/wiki/list-qc-simulators (14 November 2016).

65. 1QBit-Products, http://1qbit.com/products/ (14 November 2016).

66. QuSoft-The need for quantum software, http://www.qusoft.org/research/software/ (14 November 2016). 